\title{
Review of Manufacturing Process Defects and Their Effects on Memristive Devices
}

\author{
L. M. Bolzani Poehls ${ }^{1,5}$ (] $\cdot$ M. C. R. Fieback ${ }^{2} \cdot$ S. Hoffmann-Eifert ${ }^{3} \cdot$ T. Copetti $^{1} \cdot$ E. Brum $^{5} \cdot$ S. Menzel ${ }^{4} \cdot$ S. Hamdioui ${ }^{2}$. \\ T. Gemmeke ${ }^{1}$
}

Received: 18 December 2020 / Accepted: 3 September 2021 / Published online: 21 October 2021

(c) The Author(s) 2021

\begin{abstract}
Complementary Metal Oxide Semiconductor (CMOS) technology has been scaled down over the last forty years making possible the design of high-performance applications, following the predictions made by Gordon Moore and Robert H. Dennard in the 1970s. However, there is a growing concern that device scaling, while maintaining cost-effective production, will become infeasible below a certain feature size. In parallel, emerging applications including Internet-of-Things (IoT) and big data applications present high demands in terms of storage and computing capability, combined with challenging constraints in terms of size, power consumption and response latency. In this scenario, memristive devices have become promising candidates to complement the CMOS technology due to their CMOS manufacturing process compatibility, great scalability and high density, zero standby power consumption and their capacity to implement high density memories as well as new computing paradigms. Despite these advantages, memristive devices are also susceptible to manufacturing defects that may cause unique faulty behaviors that are not seen in CMOS, increasing significantly the complexity of test procedures. This paper provides a review about the manufacturing process of memristives devices, focusing on Valence Change Mechanism (VCM)-based memristive devices, and a comparative analysis of the CMOS and memristive device manufacturing processes. Moreover, this paper identifies possible manufacturing failure mechanisms that may affect these novel devices, completing the list of the already known mechanisms, and provides a discussion about possible faulty behaviors. Note that the identification of these mechanisms provides insights regarding the possible memristive devices' defective behaviors, enabling to derive more accurate fault models and consequently, more suitable test procedures.
\end{abstract}

Keywords Manufacturing process $\cdot$ CMOS $\cdot$ Memristive devices $\cdot$ Defects $\cdot$ Fault models

\section{Introduction}

Responsible Editor: M. Tahoori

L. M. Bolzani Poehls

poehls@ids.rwth-aachen.de

1 Chair of Integrated Digital Systems and Circuit Design (IDS), RWTH Aachen University, Germany

2 Delft, University of Technology - TU Delft, Delft, The Netherlands

3 PGI-10 \&, Forschungszentrum Jülich, JARA-FIT Jülich, Germany

4 PGI-7 \&, Forschungszentrum Jülich, JARA-FIT Jülich, Germany

5 Pontifical Catholic University of Rio Grande do SulPUCRS, School of Technology, Porto Alegre, Brazil
During the last four decades, CMOS technology miniaturized according to Moore's and Dennard's laws, which predicted the number of transistors in the same area to double every eighteen months, and as the dimensions of a device shrink, so does power consumption [29,5]. Limitations on the continued transistors' miniaturization and the increasing need for emerging applications requiring high performance systems with strict constraints already poses significant challenges to device technologies and computer architectures. Today it is possible to say that the device technology is facing the following three walls, which prevent further transistor miniaturization [12, 28]: (a) the reliability—wall associated to failure rate increase and lifetime reduction; (b) the leakage wall-meaning that the static power consumption becomes even more important than the dynamic 
power consumption, when considering the overall power consumption, and (c) the cost wall-showing that the cost per transistor via pure geometric scaling is plateauing, with no tendency to get cheaper. From the computer architecture point of view, the following walls can be identified: (a) the memory wall-due to the limited memory bandwidth that impacts performance and energy consumption of dataintensive applications as well as the growing gap between memory and processor speeds; (b) the power wall-as the practical power limit for cooling is reached and consequently, there is no possibility of further increasing CPU clock frequency; and (c) the Instruction Level Parallelism (ILP) wall-related to the always increasing complexity of keeping all cores running in parallel. The totality of these aspects limits the use of CMOS technology and von Neumann architectures as solutions for emerging applications like cognitive tasks and increases the necessity for novel devices and architectures able to deliver high performance systems. Memristive devices, quantum dots, and spin-wave devices are only some examples for emerging devices, while computation-in-memory, neuromorphic and quantum computing represent next generation's computing paradigms. In particular, memristive devices represent a promising candidate to complement the CMOS technology mainly due to their CMOS manufacturing process compatibility as well as high scalability and density [28]. However, the use of these novel devices within emerging applications depends on being able to guarantee their dependability after manufacturing. In this context it becomes crucial to properly test the fabricated devices. As already known from CMOS technology, the efficiency of test procedures depends on the understanding of manufacturing failure mechanisms, which allow the identification of possible defects and consequently, the definition of accurate fault models. Unfortunately, when considering memristive devices, the lack of information regarding manufacturing defects compromises the definition of more accurate fault models today. Thus, the main contribution of this paper is to provide a review and about the memristive device manufacturing process in order to better understand the possible defects that may affect these novel devices as well as identify the relation between manufacturing failure mechanisms and memristive devices' behavior. Further, this paper also provides some complementary insights regarding possible faults and defect injection scheme. In turn, this will guarantee the definition of accurate fault models and consequently, more efficient test procedures able to assure the device's required quality, avoiding test escapes and no trouble found components.

The paper has been organized as follows: Section 2 briefly introduces memristive devices. Section 3 provides a description of the memristive device manufacturing process taking into account some aspects regarding the CMOS manufacturing processes and possible manufacturing failure mechanisms. Section 4 provides insights regarding the possible defects that can affect novel devices followed by the presenting possible faulty behaviors. Section 5 presents the final considerations and points out open issues.

\section{Memristive Devices}

In 1971, Leon Chua postulated the fourth basic circuit element named memristive device, or memristor, while trying to establish a missing constitutive relationship between electrical charge and magnetic flux [2]. In theory, a memristive device is a passive element that can be described by the time integral of the current (charge $q$ ) through the time integral of the voltage (flux $\phi$ ) across its two terminals [2]. In other words, the memristor is a device whose resistance is called memresistance, which is a charge dependent resistance, and its value varies as a function of current and flux. A memristor has at least two distinct states, the High Resistance State (HRS) and the Low Resistance State (LRS) and can switch from HRS (LRS) to LRS (HRS) by applying a voltage $V_{\text {SET }}$ $\left(V_{\text {RESET }}\right)$ with an absolute value larger than its threshold voltage $V_{\mathrm{th}}$. The essential fingerprint of memristive devices is the pinched current-voltage (I-V) hysteresis loop, illustrated in Fig. 1a. Note that when the memristive device is floating or when the voltage $v(t)$ across the device is zero, the current $i(t)$ is also zero. The memresistance exhibits a hysteretic behavior, which can be exploited as non-volatile resistance switching memory cell [27]. Another important characteristic of memristive devices is that the pinched hysteresis loop shrinks with a higher switching frequency $f$, depicted in Fig. 1b. This originates from the highly non-linear switching kinetics which is controlled by temperature and field driven processes [37]. Figure 1c shows symbols used for representing memristive devices, where the black square represents the terminal for positive voltage switching.

In terms of classification, memristors can be initially classified in two types: (a) ionic thin film and molecular memristors, and (b) magnetic and spin-based memristor [16]. When used as a memory device, ionic thin film and molecular memristors are called resistive memories, more precisely Resistive Random Access Memories (RRAMs), being classified as a non-volatile memory [7-10]. An RRAM data storage element is a three-layer device consisting of a dielectric sandwiched between to metal electrodes. In more detail, the memory cell is based on Metal/ Insulator/Metal (MIM) structure [35]. The " $\mathrm{M}$ " in MIM denotes any reasonably good electron conductor, often asymmetric for the two sides with respect to the materials' work function and oxygen affinity, while "I" stands for insulator, often an ion or mixed conducting oxide or higher chalcogenide. RRAMs can be further classified according to the switching mode, the conductive path 


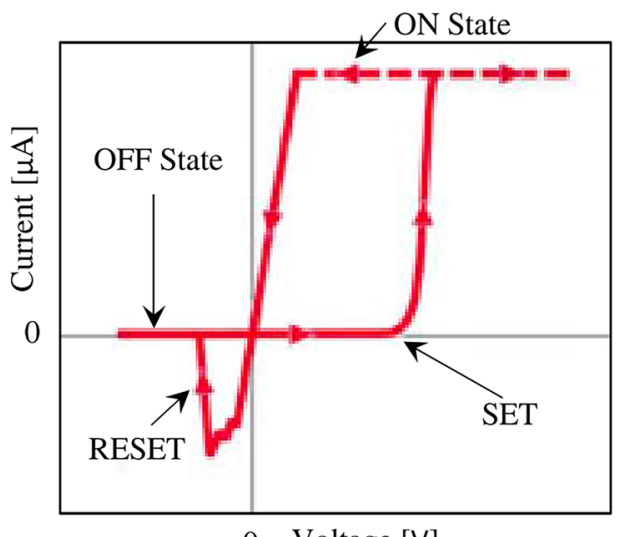

0 Voltage [V]

(a)
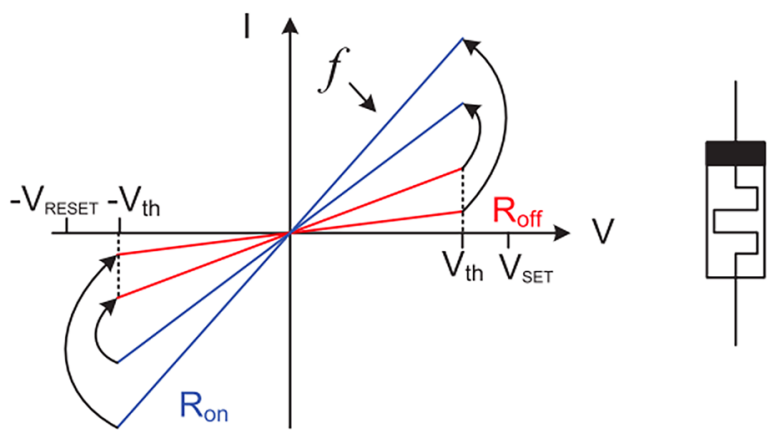

(c)

Fig. 1 (a) I-V characteristics of a biplar resistive switching device [34], (b) Influence of $f$ on I-V hysteresis loop [38], and (b) Symbols used for representing memristive devices [38]

and the switching mechanism. The switching mode can be unipolar or bipolar, where the former one depends on the voltage amplitude and consequently, SET and RESET operations are controlled by the same polarity. In contrast, bipolar switching has the SET and RESET operations controlled by reverse polarities. Moreover, RRAMs can be categorized according to the type of chemical modification responsible for the conductance change as filamentary switching and area dependent switching. When considering the filamentary switching, the Conductive Filament (CF) is formed through the electroforming process, which is a soft breakdown phenomenon that creates a locally degraded region with high defect concentration [33]. Note that the CF is made out of metallic impurities or oxygen vacancies, which are responsible for charge transport. However, in the area dependent type the switching takes place homogenously along the whole area of the electrode-oxide interface. Finally, RRAMs can be further categorized according to their switching mechanisms: (a) Valence Change Mechanism (VCM), (b) Electrochemical Mechanism (ECM) and, (c) Thermochemical Mechanism (TCM) [35]. These three classes of switching phenomena involve electro and thermochemical effects in the resistance change of a MIM memory cell [35]. The switching process of VCM cells is based on the oxygen vacancy generation and migration. The conductive path is formed due to the positively charged oxygen vacancies, while the electric current is defined by the electrostatic barrier in the band diagram. The SET operation occurs when applying negative bias voltage on the memristor active electrode of high work function material, increasing the device conductivity. However, RESET operation is performed by reversing the bias polarity, allowing the oxygen recombination. The most common VCM RRAMs use TaO, HfO and TiO.
As this work focuses on VCM devices, further information regarding the other two types of memristors can be found in [35].

\section{Manufacturing Process and Possible Defects}

This Section provides details about the manufacturing process of CMOS and memristive devices. When looking at the CMOS manufacturing process, considering most of the manufacturing steps, a certain area on the chip is masked out using the appropriate optical mask so that a desired processing step can be selectively applied to the remaining regions [9]. In more detail, the manufacturing of integrated circuits requires a large number of processes that are repeatedly performed until the circuit is completely built, first the Front-End-Of-Line (FEOL) and then the Back-End-Of-Line (BEOL). FEOL refers to the steps toward the device's development and BEOL to the steps to build the interconnections as well as any passive device required by the circuit. CMOS manufacturing process starts with the wafer preparation followed by (a) photolithography, (b) etching, (c) doping, (d) material deposition and (e) planarization. Photolithography is the process that allows creating a desired pattern on the wafer based on the following stages: (a) photoresist coating application, (b) mask alignment, (c) wafer exposure, (d) development and finally, (e) hard baking. The second step of the manufacturing process is etching, which consists of removing a portion of the material that was deposited or grown on the wafer. Usually, the etching process is performed using chemical solutions able to remove the unprotected parts, while the photoresist film defined by the photolithography acts as a protection. After removing the 
Fig. 2 (a) Memristive device manufactuirng process details and (b) Schematic view of the memristive device manufactuirng process

\begin{tabular}{|l|l|}
\hline \multicolumn{2}{|c|}{ Memristive Devices } \\
\hline Manufacturing process steps: & Possible process(es): \\
\hline 1. BE deposition & Physical Vapor Deposition (PVD) \\
\hline 2. BE patterning & Nano Imprint Lithography (NIL) or Ebeam Lithography \\
\hline 3. BE etching & Reactive lon Beam Etching (RIBE) or Reactive lon Etching (RIE) \\
\hline 4. TMO deposition & PVD or Atomic Layer Deposition (ALD) \\
\hline 5. TE deposition & PVD \\
\hline 6. TE patterning & Ebeam Lithography \\
\hline 7. TMO and TE etching & RIBE \\
\hline
\end{tabular}

(a)
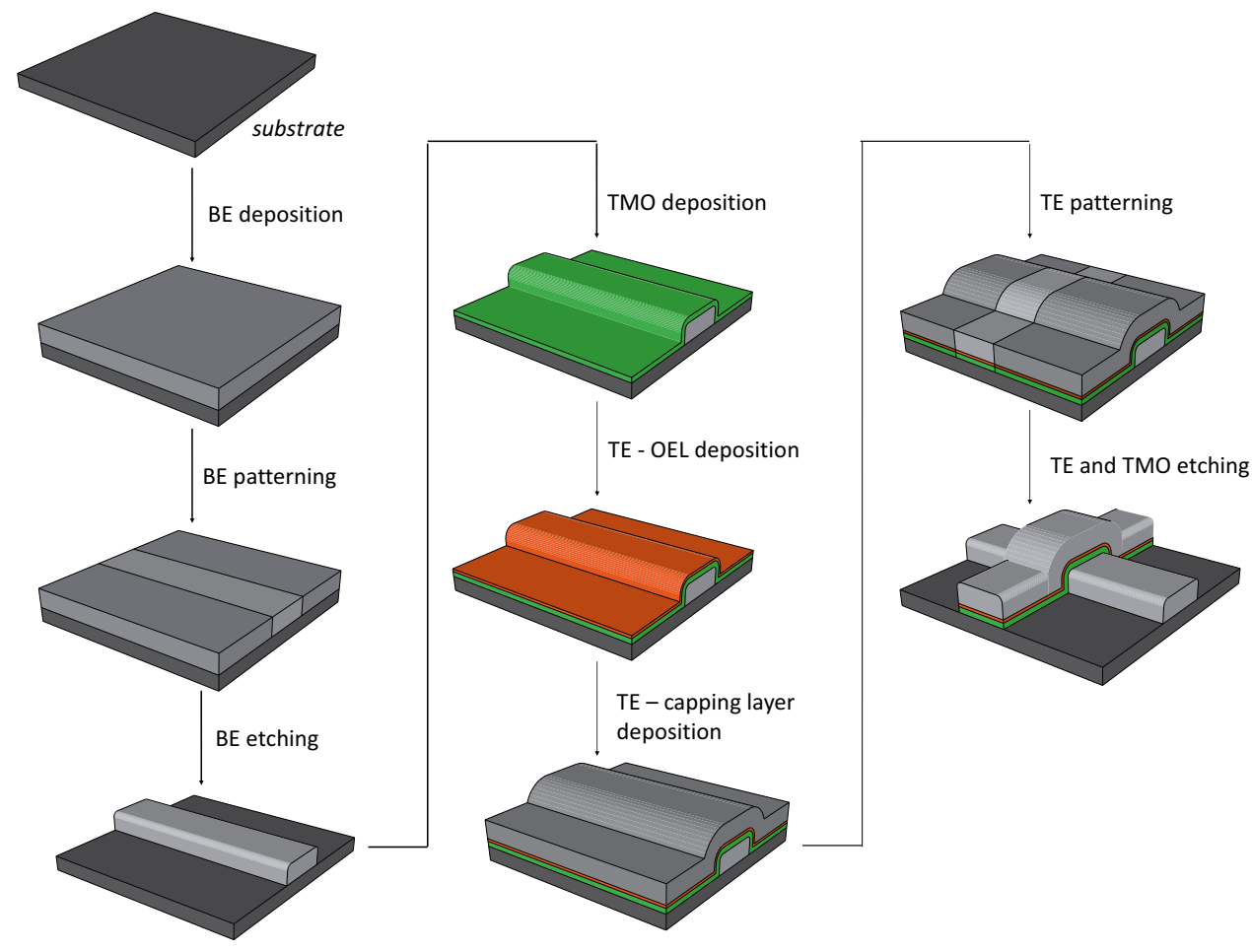

(b) unprotected material, adding atoms to the remaining material in order to modify its electrical (conduction) properties is the next step. This process is called doping. In more detail, there are two common methods for doping a material: (a) diffusion and (b) ion implantation. The next step is named deposition and is used for creating thin layers of different materials. Such depositions are always laid out over the entire wafer by adopting chemical or physical methods, known as Chemical Vapor Deposition (CVD) and Physical Vapor Deposition (PVD), respectively. Finally, the planarization is the act of obtaining a flat and smooth surface out of a rough topography, being required because the deposition of different materials results in non-uniform topographies.

As previously mentioned, filamentary, memristive VCM cells can be manufactured using different materials, being classified according to their switching mode, conductive path and switching mechanism. Different implementations of a
HfO2-based memristor (TiN/HfO2/TiN or TiN/HfO2/Ti/ TiN) are described in $[10,11,4,23]$ and a TaOx based memristor $(\mathrm{Ta} 2 \mathrm{O} / \mathrm{TaOx})$ proposed by Panasonic is described in $[15,19,15]$. The manufacturing process of memristive devices aims to create devices composed of three main parts, the Bottom Electrode (BE), the Transition Metal Oxide (TMO) and finally, the Top Electrode (TE). The memristor can be manufactured on a silicon-based substrate or on a processed integrated circuit with planarized contact pads. In general terms, the memristor fabrication includes the same basic processes, such as lithography, deposition and etching $[20,39,13,20]$. Note that the conduction mechanism in the deposited oxide relies on the bond breaking between metal and oxygen ions. Hence, an essential aspect regarding the manufacturing of memristors is that the process typically does not require doping, which eliminates a relevant source of process variation in standard CMOS circuits [25, 24]. 
For this study, mirco- and nanosized crossbar-type oxidebased memristive devices are taken as one prominent example [26]. Here, memristive cells are built at the lines' cross junctions. Replacing the electrode lines of a single device by word- and bit-lines of an array, it is easily anticipated that the single crossbar elements can be considered as building blocks of passive memristive crossbar arrays [21, 22]. A slightly different device form is the pillar cell, where the MIM layer stack is deposited as a whole and is finally etched into the desired shape. The BE and TE are contacted by vias [11]. Note that in this paper, the focus is laid on the crossbar cell design due to the possibility to build on every substrate, either a passive, planar disc or a CMOS-type substrate with contact pads reaching to the top surface [10]. It is important to highlight that after manufacturing, especially the oxidebased filamentary-type devices, usually have a very high electrical resistance and a large voltage is required for the very first SET operation, also known as the forming process [16]. This process, a controlled soft breakdown, drastically reduces the device resistance allowing the resistance switching behavior in the subsequent cycles for the filamentary regime. Figure 2a summarizes the main manufacturing process steps for a typical crossbar structured VCM-based memristive device $[20,39,13,20]$, including the possible processes, whereas Fig. $2 b$ depicts the schematic view of the entire manufacturing process step-by-step.

Note that it is possible to adopt different processes, depending on the material used for fabricating the devices. For example, the etching of the BE, which is a high work function metal can be performed by Reactive Ion Etching (RIE), when using TiN, or Reactive Ion Beam Etching (RIBE), when adopting $P t$ as material. The BE is the first element fabricated, including the material deposition, patterning and etching. The second part to be manufactured is the TMO, the transition metal oxide, i.e., the regime where the resistive switching takes place, and which can be made from either single or double layers of metal oxides. The TE is composed of two different layers, the Oxygen Exchange Layer (OEL), which is formed from a low barrier, chemically active metal and a capping layer, which protects the reactive metal against atmosphere or further chemical process steps, as depicted in Fig. 2b. The TE is deposited and the patterning and etching are performed for all deposited layers, TMO and TE. Finally, after the structuring of the full device a passivation step can be performed.

Defining an accurate fault model for memristive devices based on realistic manufacturing defects represents a big challenge because the access to real data collected during a step-by-step inspection of manufactured devices is limited. In a first approach given in the literature, as adopted for CMOS technology, it is assumed that possible manufacturing defects can be modeled using linear resistances in order to identify the faults affecting memristive devices [34, 34].
In more detail, the defect injection scheme is based on the introduction of resistors connected in series with the memristive device. However, as shown in literature, the use of linear resistance to model the defects within RRAM at the terminals is inaccurate, as the resistance cannot properly reflect how defects impact the non-linearity (loop hysteresis) of the RRAM device [7]. In more detail, this approach can provide some insights regarding the possible faults, but it lacks accuracy. Analyzing the memristive device manufacturing process may allow to derive a more suitable defect injection mechanism and more accurate fault models. Table 1 summarizes the possible defects that can occur during the manufacturing of the analyzed novel crossbar-like memristive devices and their associated misbehaviors.

The possible defects included in Table 1 were derived from experience with the VCM-based memristive device manufacturing process, the continuum and compact models for filamentary switching, bipolar VCM-type resistance switching [36], and literature associated to CMOS technology as well to memristive devices as discussed in the following.

As previously mentioned, crossbar memristive device cells can be fabricated after FEOL, more precisely after the fabrication of the lower metal layers of the BEOL. The standard CMOS fabrication process (FEOL and BEOL) introduces defects that often are caused by impurity depositions, behaving as resistive defects at electrical level. Resistive defects represent the lumped effect of broken or irregular shaped metal lines, narrow, cracked or non-existent vias, and dust particles deposited between layers impeding proper electric conductivity [14]. The fabrication of lower metal layers adopts the standard CMOS process and consequently, the same defects may occur. Potential defects include incomplete wiring or via fills and thinner wires, which result in resistive opens, shorts and bridges caused by line-edge roughness or misalignment, for example [34]. As previously mentioned, the devices adopted for the analysis proposed in this paper can be manufactured on different substrate types, making a further investigation about the possible defects that can occur in this part of the circuit or even in the interface with the memristive devices essential. Note that pillar cells are fabricated following different manufacturing process steps and consequently, may be affected yet by other defects not to be discussed in this paper.

When considering the fabrication of VCM-based memristive devices, chemical and physical conditions can affect the composition as well as the microstructure of the deposited BE thin film and imprint residual stress, which in turn affects the quality of the forming process [14]. In more detail, the amplitude of the signal required for forming affects the LRS value. In extreme situations, this effect can prevent the forming process entirely, resulting in an open circuit-like behavior. BE deposition can also be contaminated by precursors, 
Table 1 VCM-based filamentary, binary-type memristive devices based on TMO resistive switching layers: possible defects and faulty behaviors

\begin{tabular}{|c|c|c|}
\hline Manufacturing process steps: & Possible defects: & Possible defective/faulty behavior(s): \\
\hline \multirow{3}{*}{ BE deposition } & Thickness variations (thinner of thicker) & \multirow{3}{*}{$\begin{array}{l}\text { Increased contact/line resistance; lower or higher heat conductance; } \\
\text { lower or higher quality of forming process }\end{array}$} \\
\hline & Poor or no bonding with contacts & \\
\hline & Contamination associated to depostion process & \\
\hline \multirow{4}{*}{ BE patterning } & Contamination by residual photolayer/polymer & \multirow{4}{*}{$\begin{array}{l}\text { Increased resistance; lower heat conductance; undefined impurities on } \\
\text { the BE surface; line length/resistance modified; switching area modified; } \\
\text { well area undefined/high sheet resistance in the regime of reduced BE } \\
\text { thickness }\end{array}$} \\
\hline & Shift of the structure (markers) & \\
\hline & Over-/under-development & \\
\hline & Trapezoidal shape of the resist structure & \\
\hline \multirow{4}{*}{ BE etching } & Under etching (shorts and bridges) & \multirow{4}{*}{$\begin{array}{l}\text { Increased line resistance; increased capacitance; change in the } \\
\text { electrode work function; electrical field variation (hot spots) }\end{array}$} \\
\hline & Overetching (opens) & \\
\hline & Contamination by etchant & \\
\hline & Higher electrode roughness & \\
\hline \multirow{5}{*}{ TMO deposition } & Poor bonding with $\mathrm{BE}$ & \multirow{5}{*}{$\begin{array}{l}\text { Increased variability of I-V characteristics in the pristine state; increased } \\
\text { variability of the electroforming voltage; inreased variability of the } \\
\text { resistance; reduced switching stability; uncontrolled increase/decrease } \\
\text { of the initial leakage; heat conductance modification; oxygen vacancy } \\
\text { concentration variations }\end{array}$} \\
\hline & $\begin{array}{l}\text { TMO local composition variations in the metal to } \\
\text { oxygen stoichiometry }\end{array}$ & \\
\hline & TMO thickness variations & \\
\hline & Contamination by carbon or hydroxy-impurities & \\
\hline & Crystal structure variations & \\
\hline \multirow{3}{*}{$\begin{array}{l}\text { TE deposition (including OEL } \\
\text { and capping layer) }\end{array}$} & Thickness variations & \multirow{3}{*}{$\begin{array}{l}\text { Electroforming voltage variations; oxygen chemical potention between } \\
\text { OEL and TMO variation; increased resistance; increased diffusion path; } \\
\text { increased variability }\end{array}$} \\
\hline & Contamination associated to depostion process & \\
\hline & Poor bonding between OEL and capping layer & \\
\hline TE patterning & Contamination by residual photolayer/polymer & Increased contact resistance \\
\hline \multirow{2}{*}{ TE etching } & Sidewall redeposition & \multirow{2}{*}{ Shorts; edge modficaiton or damage; weak spots for filament formation } \\
\hline & Etching damage & \\
\hline \multirow{2}{*}{$\begin{array}{c}\text { Passivation \& contact pad } \\
\text { opening }\end{array}$} & Poor or no bonding with contacts & \multirow{2}{*}{ Increased or reduced resistance; lower or higher heat conductance } \\
\hline & Contamination & \\
\hline
\end{tabular}

materials related to previous production steps, dust/small particles, etc. Moreover, it is possible to observe BE thickness variations as well as poor or no bonding between $\mathrm{BE}$ and pads. These possible defects can cause memristive devices' deviations, increasing their resistance as well as reducing their heat conductance and the forming process quality. BE patterning can also be contaminated by residual photolayer/polymer, have over-/under-development and a modification of the resist structure shape. These possible defects may lead to increased resistance, lower heat conductance and variation of the switching area. BE etching could leave the metal surface rough, leading to large resistance variations. Under- and over-etching can introduce open, short and bridge defects, as observed in CMOS technology. Moreover, BE defects can also change the electrode work function and introduce variation of the electrical field (hot spots). Resuming, BE patterning and etching may lead to switching area variations, affecting the device performance $[30,32]$. In more detail, the device's size affects its resistance because it alters the probability of forming the CF [10]. The TOM deposition is vulnerable to different problems related to precursors and cleaners, which may introduce thick or thin localized spots [34, 34]. Other important possible defects associated to TMO deposition include poor bonding with $\mathrm{BE}$ and variations in the crystal structure. The oxygen vacancies concentration and the oxide thickness have a strong impact on the device's performance [34]. Note that during the deposition process of TMO, an Oxygen Exchange Layer (OEL) and a capping layer are added. Further, a deficient capping layer deposition can lead to large variations in the characteristics of the forming process and the efficiency of the switching behavior [14]. The TE is fabricated on the top of the capping layer following a similar process as the BE. The etching step may lead to material redeposition along the device's sidewalls. This material can form a leakage path next to the VCM-based memristive device reducing the resistance and the forming voltage and increasing the device's variability $[1,1]$. After manufacturing the TE, the memristor is pacified, which means that the device is isolated from the surroundings and the TE is connected to the metal layers. Finally, a further step is still required in order to have the manufactured device working. In more detail, the $\mathrm{CF}$ in the oxide has to be formed. The forming step may be performed by applying different schemes, most commonly voltage pulses with different pulse widths. Note that the current that flows through the memristor during the forming step has a severe impact on the CF shape, which in turn influences the device characteristics during operation [34]. The forming current needs to be kept constant, since variations will result in more resistive variations later on $[31,18]$. Higher forming currents result in wider CF structures, leading to lower mean resistance and standard deviation, while lower currents generally lead to higher mean and standard deviation of the device resistance [34]. Thus, two different defects can occur during the forming step. The first one is an oxide breakdown of the memristor and the second occurs when the forming step fails, and no CF is formed. As result of the first defect, the device remains always in LRS, or if 
less severe, the device is still able to switch, but the resistance has shifted below its intended value. However, when considering the second possible defect, the device is stuck in HRS.

\section{Discussion about Memristive Device Fault Models}

The development of efficient manufacturing test procedures requires realistic defect injection schemes and accurate fault models. From the previous Section it is possible to identify that manufacturing defects can affect different memristor's parameters causing the following main misbehaviors:

- Increased or decreased resistance of contacts and lines;

- Lower heat conductance;

- Lower quality or ineffective forming step;

- Increased BE-oxide interface oxygen vacancy concentration;

- Changes in oxygen vacancy concentration in the oxide.

The increasing or decreasing resistance of the contacts and lines may be modeled using a linear resistor, the same defect injection scheme adopted for CMOS technology. However, this defective behavior will be propagated as an analog deviation, which may require more complex test procedures, such as parametric test strategies.

The deviation associated to the heat conductance could affect the switching properties of the device. The stochastic properties of the switching behavior will significantly increase the complexity of properly model this misbehavior as well as test strategies. With respect to the possible faulty behaviors, it would be possible to identify four different situations: (a) the device is not able to correctly switch during the defined SET or RESET time, impacting the device's functionality, (b) the device presents parametric deviations, impacting its performance, (c) the device presents a random misbehavior, being observed in some occasions, and (d) the device's reliability is affected due to the degradation of the switching capacity over time caused by aging mechanisms. Note that the first situation represents Easy-To-Detect (ETD) faults, since the faulty behavior will be propagated at logic level, making its detection trivial [6]. However, the second and third situation represents Hard-To-Detect (HTD) faults that do not impact on the device's functionality, but can cause parametric deviations, increasing test complexity and requiring the use of e.g. defect-oriented test strategies. Note that HTD faults do not always lead to incorrect behavior; nevertheless, they compromise the device's reliability. Extensive studies including the impact of temperature on the behavior of memristive devices should be conducted to allow an accurate identification of the relation between heat and memristor's behavior.

Regarding the third point of the possible misbehaviors, the correct formation of the $C F$ during the forming process is considered critical as it may lead to defective devices. Defective devices may remain in HRS, which leads to ETD faults, more precisely to stuck-at faults. However, there is also a possibility that devices may switch from HRS or even LRS to an undefined state. The detection of undefined states is not trivial, since it demands the measurement of analog values/states. To better illustrate this situation, electrical simulations of a RRAM $(3 \times 3$ word cell array $)$ with

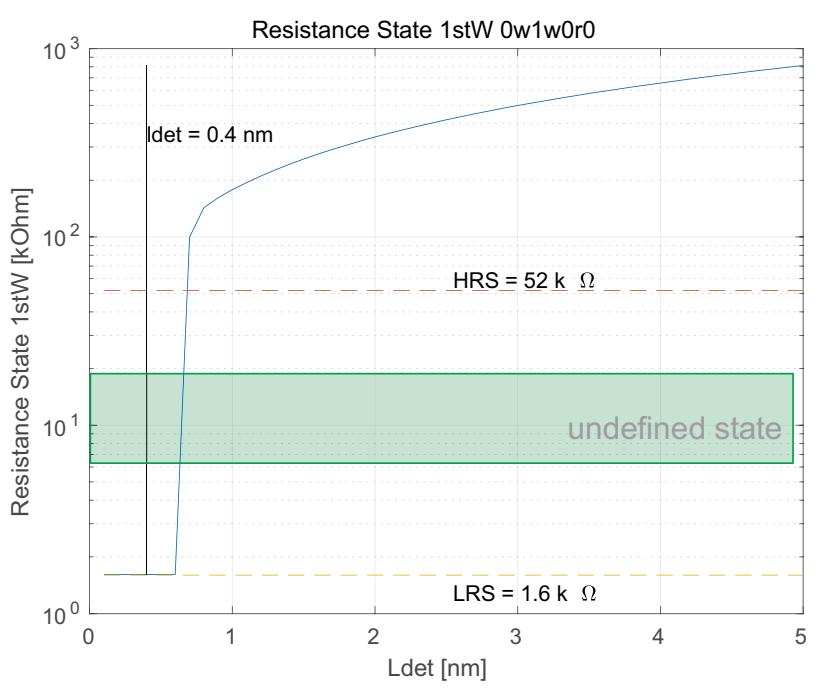

(a)

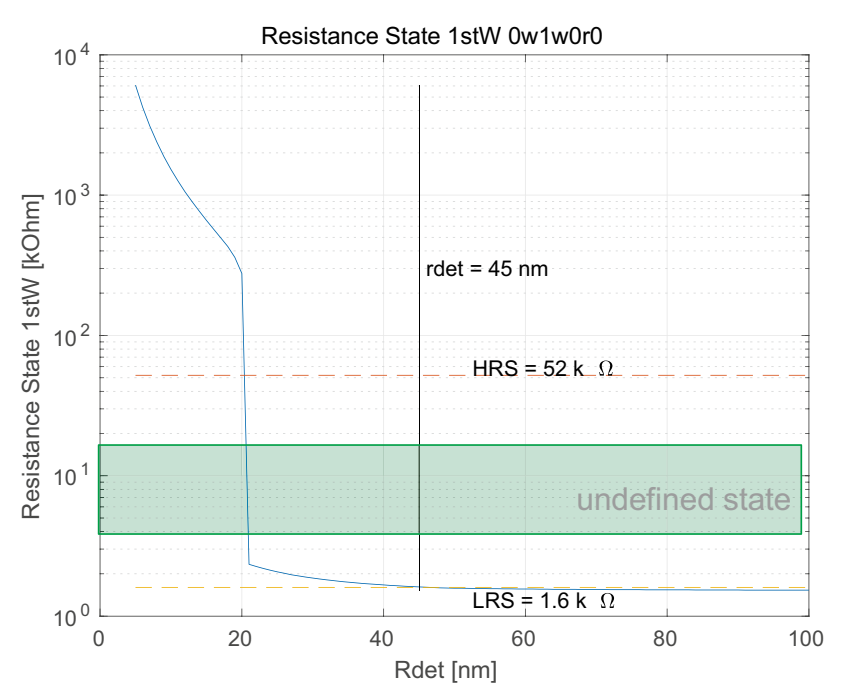

(b)

Fig. 3 (a) Ldet variation impact on resistance state of the memristor, and (b) Rdet impact of tne resistance state of the memristor 
peripheral circuitry were performed. The memory array was implemented using the $130 \mathrm{~nm}$ Predictive Technology Model (PTM) for the CMOS-based circuits and the RRAM (Pt/HfO2/TiOx/Pt) compact model from [17]. The voltage adopted for performing a write ' 1 'operation, or in other words a SET operation, is equal to $1.6 \mathrm{~V}$. The RESET operation is performed by applying a voltage of $-1.7 \mathrm{~V}$, and READ operations require voltage pulses of $0.16 \mathrm{~V}$. The forming step has a strong impact on the length (Ldet) and radius (Rdet) of the CF. For example, higher forming current will lead to wider and longer filaments, which have lower resistance, and vice versa. Figure 3 depicts the impact of varying these two different parameters associated to the $\mathrm{CF}$. In Fig. 3a it is possible to see the impact of varying (Ldet), and in Fig. 3b Rdet [17, 3]. The resistance state values of the memristor were measured after performing the SET operation present in the operating sequence shown in Fig. 3a and $b$. Moreover, the range intervals adopted for performing these simulations were defined based on the constraints defined in [17], where the nominal value for Ldet is $0.4 \mathrm{~nm}$ and for Rdet is $45 \mathrm{~nm}$.

Observing Fig. 3a, it is possible to see a linear dependency between Ldet and the resistance state of the memristive device. The resistance state of the memristor changes from $1.6 \mathrm{k} \Omega$ to nearly $100 \mathrm{~K} \Omega$ when Ldet assumes a value higher than $0.6 \mathrm{~nm}$, showing that the memristor is not able to properly switch from ' 0 ' to ' 1 ', SET operation. Figure $3 b$ illustrates the relation between Rdet and resistance state of the memristor. The graph shows that $R$ det needs to be larger than $20 \mathrm{~nm}$ to guarantee the correct execution of the write ' 1 ' operation, otherwise the memristor would remain at logic ' 0 ' (HRS). Thus, these results demonstrate that variation on the manufacturing parameters of the memristive devices, in this case more specifically to the forming step (length and radius of the $\mathrm{CF}$ ), can compromise the correct behavior of the novel device. Further, the results plotted in these two graphs show that depending on the Ldet and Rdet values the device can assume an undefined state, since it reaches a value in between LRS and HRS, which can complicate the fault detection during the execution of manufacturing test procedures.

The defective behavior associated to increased BE-oxide interface oxygen vacancy concentration may affect the switching properties and the device resistance. Figure 4 depicts the memristor devices' behavior when changing the oxygen vacancy concentration, minimum (Ndiscmin) and maximum (Ndiscmax) [17]. Figure 4 depicts the resistance state of the cell when performing the second write operation related to the operation sequence $0 w 1 w 0 r 0$. In Fig. $4 \mathrm{a}$, assuming an increment factor of 0.001 for Ndiscmin, it is possible to see that cell's correct behavior may only be guaranteed by keeping Ndiscmin smaller than $0.4 \times 10^{26} \mathrm{~m}^{-3}$, otherwise, the cell remains at LRS or even switches to an undefined state. When varying Ndiscmax from $10 \times 10^{26} \mathrm{~m}^{-3}$ to $30 \times 10^{26} \mathrm{~m}^{-3}$, assuming an increment factor of $1 \times 10^{26} \mathrm{~m}^{-3}$ during each simulation, the resistance state remains relatively constant, around $52 \mathrm{k} \Omega$, when considering an Ndiscmax below $20 \times 10^{26} \mathrm{~m}^{-3}$. Note that from $20 \times 10^{26} \mathrm{~m}^{-3}$ to $22 \times 10^{26} \mathrm{~m}^{-3}$, the resistance state gradually decreases until reaching values close to LRS, which means that the cell is going to be stuck-at-1. Thus, from Fig. 4a it becomes clear that the minimal oxygen vacancy concentration has an exponential effect on the HRS resistance. Hence, if the manufacturing process suffers from the last two main misbehaviors listed at the beginning of this Section, more and more faults leading to undefined states will be observed.

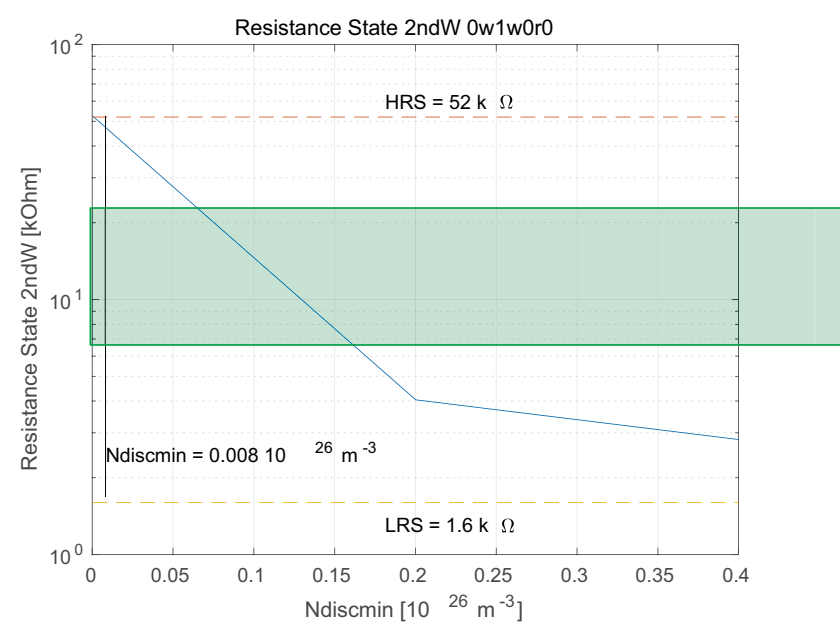

(a)

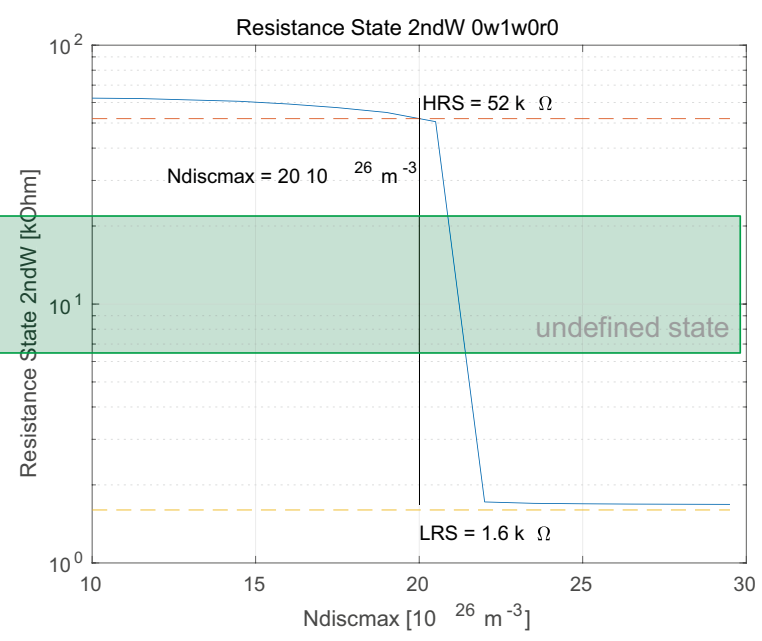

(b)

Fig. 4 (a) Ndiscmin variation impact on resistance state of the memristor, and (b) Ndiscmax impact of tne resistance state of the memristor 
Read faults are also be observed more frequently, reducing the reliability of the memristor-based cells. Moreover, lower HRSs will be observed, which increase the energy consumption.

In general, more vacancies lead to faster switching and lower forming voltage. The latter may lead to oxide breakdown or even to different faults associated to READ operations, as observed in the CMOS technology. Changes in oxygen vacancy concentration inside the oxide may also affect the quality of the forming process, resulting in similar fault behavior as the previous defective behavior (lower quality or ineffective forming step). In general terms, these faulty behaviors can be observed at chemical and electrical level and, in order to derive an accurate fault model, need to be properly represented by a realistic defect injection scheme. Clearly, a linear resistor can not properly represent these defects because it affects the surroundings of the memristor only and not the internal state itself. One possibility for addressing this issue could be based on the directly modification of manufacturing parameters presents in the description of resistive device models, such as the models described in [17].

As observed in nanoscale CMOS technology, the expected defects do not necessarily propagate faults at logic level, hence deviations may be observed at electrical level only. The presence of parametric faults will require the adoption of not only software-based test approaches, able to detect functional faults at logic level, but also the adoption of hardware-based strategies able to perform parametric testing. Another important point that increases the test complexity when considering memristive devices is related to the fact that manufacturing defects can cause intermittent and random faults [8]. In [34] the authors defined two distinct fault models for RRAMs: (1) the conventional fault model and (2) the unique fault model. In more detail, the conventional fault model is composed of faults that are similar to the ones observed in traditional memories, while unique fault models represent a set of faults that emerge due to RRAMs specific nature. Thus, according to the information above, manufacturing defects affecting memristive devices tend to introduce HTD faults. This is due to the stochastic and analog nature of the device. Consequently, the development of test procedures becomes more complex and expensive, since either the adoption of hardware-based strategies is required in order to perform parametric testing or special test conditions need to be defined.

\section{Final Considerations}

The development of efficient manufacturing test procedures represents a critical aspect limiting the adoption of memristive devices for implementing emerging applications, since test escapes may directly affect the reliability of the entire application. Despite the similarities of the memristive device manufacturing process with the one adopted for CMOS devices, the possible defects affecting such novel devices may lead to specific faulty behaviors. As previously mentioned, the currently knowledge regarding these topics indicates that defective memristive devices cause different faulty behaviors, when comparing to the possible faults observed in the CMOS technology. These unique functional or electrical deviations occur due to the stochastic and analog nature of the memristive device, making the definition of a specific and realistic fault model and the respective defect injection scheme crucial. For properly addressing this challenge, companies and laboratories that are able to fabricate memristive devices should provide more information about their manufacturing processes. In more detail, a deep inspection strategy should be applied in order to accurately measure the functional and electrical deviations associated to each manufacturing process step. The results obtained by performing this kind of inspection could be used for identifying and characterizing the most common and critical defects, which in turn would allow the definition of a more realistic defect injection scheme and consequently, the identification of more accurate fault models. As future work, we intend to analyze the manufacturing process of memristive devices adopted at Research Center Jülich (FZJ), Germany, and define an manufacturing inspection strategy. The results obtained will be used for deriving a more accurate fault model, making the development of more suitable test procedures, able to deal with the unique memristive device nature, possible.

Acknowledgments This work was mainly supported by Exploratory research space @ RWTH Aachen for interdisciplinary Seed Fund Projects - thematic call: Neuro-Inspired Computing (neuroIC) within the Silicon Synapse (SisSy) project and by the Federal Ministry of Education and Research (BMBF, Germany) within the NEUROTEC project (project numbers 16ES1134 and 16ES1133K).

Funding Open Access funding enabled and organized by Projekt DEAL.

Data Availability The datasets generated during and/or analysed during the current study are available from the corresponding author on reasonable request.

Open Access This article is licensed under a Creative Commons Attribution 4.0 International License, which permits use, sharing, adaptation, distribution and reproduction in any medium or format, as long as you give appropriate credit to the original author(s) and the source, provide a link to the Creative Commons licence, and indicate if changes were made. The images or other third party material in this article are included in the article's Creative Commons licence, unless indicated otherwise in a credit line to the material. If material is not included in the article's Creative Commons licence and your intended use is not permitted by statutory regulation or exceeds the permitted 
use, you will need to obtain permission directly from the copyright holder. To view a copy of this licence, visit http://creativecommons. org/licenses/by/4.0/.

\section{References}

1. Beckmann K, Holt J, Olin-Ammentorp W, Alamgir Z, Van Nostrand J, Cady NC (2017) The effect of reactive ion etch (\{RIE $\}$ ) process conditions on ReRAM device performance. Semicond Sci Technol 32(9):95013. https://doi.org/10.1088/1361-6641/aa7eed

2. Chua L (1971) "Memristor - The Missing Current Element," IEEE Trans. Circuit Theory. CT-18:5

3. Cüppers F et al (2019) "Exploiting the switching dynamics of HfO2-based ReRAM devices for reliable analog memristive behavior". APL Mater 7(9). https://doi.org/10.1063/1.5108654

4. Dalgaty T et al (2019) "Hybrid neuromorphic circuits exploiting nonconventional properties of RRAM for massively parallel local plasticity mechanisms". APL Mater 7:8. https://doi.org/10.1063/1.5108663

5. Dennard R, Gaensslen F, Yu W-N, Rideout L, Bassous E, Le Blanc A (1974) Design of Ion-Implanted Small MOSFET' $S$ Dimensions with Very. IEEE J Solid State Circuits 9(5):257-268

6. Fieback M et al (2019) "Device-aware test: A new test approach towards DPPB level," in Proceedings - International Test Conference. 2019. https://doi.org/10.1109/ITC44170.2019.9000134

7. Fieback M, Taouil M, Hamdioui S (2019) "Testing resistive memories: Where are we and what is missing?," in Proceedings - International Test Conference. 2018. https://doi.org/10.1109/ TEST.2018.8624895

8. Fieback M, Medeiros GC, Gebregiorgis A, Aziza H, Taouil M, Hamdioui S (2021) Intermittent Undefined State Fault in RRAMs. IEEE European Test Symposium (ETS) 2021:1-6. https://doi.org/ 10.1109/ETS50041.2021.9465401

9. García Gervacio J, Champac V (2018) Timing Performance of Nanometer Digital Circuits Under Process Variations.

10. Govoreanu B et al " 31.6 10x10nm² Hf/HfOx Crossbar Resistive RAM with Excellent Performance, Reliability and Low-Energy Operation".

11. Grossi A et al (2018) Cell-to-Cell Fundamental Variability Limits Investigation in OxRRAM Arrays. IEEE Electron Device Lett 39(1):27-30. https://doi.org/10.1109/LED.2017.2774604

12. Hamdioui S et al (2017) "Memristor for computing: Myth or reality?," in Proceedings of the 2017 Design, Automation and Test in Europe, DATE 2017 722-731. https://doi.org/10.23919/DATE. 2017.7927083

13. Hardtdegen A, La Torre C, Cuppers F, Menzel S, Waser R, HoffmannEifert S (2018) Improved switching stability and the effect of an internal series resistor in HfO2/TiOx Bilayer ReRAM Cells. IEEE Trans Electron Devices 65(8):3229-3236. https://doi.org/10.1109/TED. 2018.2849872

14. Haron NZ, Hamdioui S (2011) "On defect oriented testing for hybrid CMOS/memristor memory," in Proceedings of the Asian Test Symposium. 353-358. https://doi.org/10.1109/ATS.2011.66

15. Hayakawa $\mathrm{Y}$ et al (2015) "Highly reliable $\mathrm{TaO} \times \mathrm{ReRAM}$ with centralized filament for 28-nm embedded application". IEEE Symp VLSI Circuits Dig Tech Pap 2015(2011):T14-T15. https:// doi.org/10.1109/VLSIC.2015.7231381

16. Ielmini D, Milo V (2017) Physics-based modeling approaches of resistive switching devices for memory and in-memory computing applications. J Comput Electron 16(4):1121-1143. https://doi.org/ $10.1007 / \mathrm{s} 10825-017-1101-9$

17. Jart vcm v1b (2011) http://www.emrl.de/JART.html, Accessed: 2021-04-11
18. Kalantarian A et al (2012) "Controlling uniformity of RRAM characteristics through the forming process". IEEE Int Reliab Phys Symp Proc 3-7. https://doi.org/10.1109/IRPS.2012.6241874

19. Kawahara A et al (2012) "An $8 \mathrm{Mb}$ multi-layered cross-point ReRAM macro with $443 \mathrm{MB} / \mathrm{s}$ write throughput". Dig Tech Pap IEEE Int Solid-State Circuits Conf 55:432-433. https://doi.org/ 10.1109/ISSCC.2012.6177078

20. Kim W, Menzel S, Wouters DJ, Waser R, Rana V (2016) 3-Bit Multilevel Switching by Deep Reset Phenomenon in Pt/W/TaOX/ Pt-ReRAM Devices. IEEE Electron Device Lett 37(5):564-567. https://doi.org/10.1109/LED.2016.2542879

21. Kügeler C, Meier M, Rosezin R, Gilles S, Waser R (2009) High density 3D memory architecture based on the resistive switching effect. Solid State Electron 53(12):1287-1292. https://doi.org/10. 1016/j.sse.2009.09.034

22. Kügeler C, Rosezin R, Linn E, Bruchhaus R, Waser R (2011) Materials, technologies, and circuit concepts for nanocrossbar-based bipolar RRAM. Appl Phys A Mater Sci Process 102(4):791-809. https://doi.org/10.1007/s00339-011-6287-2

23. Kügeler C, Zhang J, Hoffmann-Eifert S, Kim SK, Waser R (2011) "Nanostructured resistive memory cells based on 8-nmthin TiO2 films deposited by atomic layer deposition". J Vac Sci Technol B Nanotechnol Microelectron Mater Process Meas Phenom 29(1):01AD01. https://doi.org/10.1116/1.3536487

24. Kuhn K (2008) "Managing Process Variation in Intel's 45nm CMOS Technology".

25. Kuhn KJ et al (2011) Process Technology Variation. IEEE Trans Electron Devices 58(8):2197-2208. https://doi.org/10.1109/ TED.2011.2121913

26. Li C et al (2018) Analogue signal and image processing with large memristor crossbars. Nat Electron 1(1):52-59. https://doi. org/10.1038/s41928-017-0002-z

27. Mathur ND (2008) The fourth circuit element. Nature 455(7217):7437. https://doi.org/10.1038/nature07437

28. Mazumder P, Kang SM, Waser R (2012) Memristors: Devices, models, and applications. Proc IEEE 100(6):1911-1919. https:// doi.org/10.1109/JPROC.2012.2190812

29. Moore GM (1965) "Cramming more components onto integrated circuits with unit cost," Electronics. 38(8):114. [Online]. Available: https://newsroom.intel.com/wp-content/ uploads/sites/11/2018/05/moores-law-electronics.pdf

30. Niu D, Chen Y, Xu C, Xie Y (2010) "Impact of process variations on emerging memristor". Proc - Des Autom Conf 877882. https://doi.org/10.1145/1837274.1837495

31. Nguyen C et al (2017) "Study of forming impact on 4Kbit RRAM array performances and reliability", 2017 IEEE 9th Int. Mem Work IMW 2017:4-7. https://doi.org/10.1109/IMW.2017.7939105

32. Nickel JH et al (2013) Memristor structures for high scalability: Non-linear and symmetric devices utilizing fabrication friendly materials and processes. Microelectron Eng 103:66-69. https:// doi.org/10.1016/j.mee.2012.09.007

33. Vatajelu EI, Aziza H, Zambelli C (2015) "Nonvolatile memories: Present and future challenges," in Proceedings of 2014 9th International Design and Test Symposium, IDT 2014. 61-66. https://doi.org/10.1109/IDT.2014.7038588

34. Vatajelu EI, Prinetto P, Taouil M, Hamdioui S (2017) "Challenges and solutions in emerging memory testing". IEEE Trans Emerg Top Comput 1-1. https://doi.org/10.1109/tetc. 2017.2691263

35. Waser R (2008) "Electrochemical and thermochemical memories,". https://doi.org/10.1109/IEDM.2008.4796675

36. Waser R (2020) "JART - Jülich Aachen Resistive Switching Tools". http://www.emrl.de/JART.html (accessed Dec. 19, 2020)

37. Waser R, Dittmann R, Staikov C, Szot K (2009) Redox-based resistive switching memories nanoionic mechanisms, prospects, 
and challenges. Adv Mater 21(25-26):2632-2663. https://doi. org/10.1002/adma.200900375

38. Yu J, Du Nguyen HA, Xie L, Taouil M, Hamdioui S (2019) "Memristive Devices for Computation-In-Memory,". https://doi. org/10.23919/DATE.2018.8342278

39. Zhang $\mathrm{H}$ et al (2018) Understanding the Coexistence of Two Bipolar Resistive Switching Modes with Opposite Polarity in Pt/TiO2/Ti/Pt Nanosized ReRAM Devices. ACS Appl Mater Interfaces 10(35):29766-29778. https://doi.org/10.1021/acsami. 8 b09068

Publisher's Note Springer Nature remains neutral with regard to jurisdictional claims in published maps and institutional affiliations.

L. M. Bolzani Poehls graduated in Computer Science at the Federal University of Pelotas (Brazil) in 2001 and received the best thesis award for her work. In the year 2004 she received her Master of Science Degree in Electrical Engineering at Pontifical Catholic University of Rio Grande do Sul (Brazil). During her Ph.D. her work was focused on the development of New Techniques for Highly Reliable Systemson-Chip. In 2008 she received her Ph.D. in Computer Engineering from the Politecnico di Torino (Italy). She holds three postdoctoral titles, the first one accomplished in 2008 in the field of Low Power Design of Integrated Circuits (ICs) at the Politecnico di Torino, the second one in 2010 with focus on Electromagnetic Inteference-Aware Systems-onChip Design at the Catholic University of Rio Grande do Sul (Brazil), and the third form the Politecnico di Torino (Italy) achieved in 2013 in the area of emerging technologies. She is Professor of the Engineering Faculty of the Catholic University of Rio Grande do Sul and part of the EASE research laboratory, leading the OASiS research group. At the moment she is a senior researcher at RWTH Aachen University working on test and reliability of memristive devices, more specifically she is working developing new fault models and manufacturing testing strategies for Resistive RAMs. Her fields of interest basically include: Fault Tolerance and Testing of Systems-on Chip, Power-, Aging- and Temperature-Aware Integrated Circuits Design and Development of Electronic Design Automation (EDA) tools for optimization of Integrated Circuits, and ultimately emerging technologies. Among other activities, she continues to serve as technical committee member in many IEEE-sponsored conferences. She is member of the IEEE Latin American Test Symposium Steering Committee and Coordinating Editor of Journal of Electronic Testing: Theory and Application.

M. C. R. Fieback received the B.Sc. and M.Sc. degrees from the Delft University of Technology, The Netherlands, in 2015 and 2017, respectively, where he is currently pursuing the Ph.D. degree. His research interests include device and defect modeling, and test and reliability of emerging memories.

S. Hoffmann-Eifert received the Diploma degree in physics and the $\mathrm{Ph} . \mathrm{D}$. degree in electrical engineering from RWTH Aachen University, Aachen, Germany, in 1992 and 1998, respectively. She is currently a Senior Researcher with Peter-Grünberg-Institut 7, Forschungszentrum Jülich
$\mathrm{GmbH}$, Jülich, Germany, where she is leading the Atomic Layer Deposition Group.

T. Copetti graduated in 2012 and received his Master of Science Degree in 2015, both in Electrical Engineering at Pontifical Catholic University of Rio Grande do Sul (PUCRS), Brazil. Recently, in 2020 he did his thesis defense at the Federal University of Rio Grande do Sul (UFRGS). At the moment he starts as a Postdoc at the Chair of Integrated Digital Systems and Circuit Design (IDS) at RWTH University, Germany. His fields of interest include Fault Tolerant Integrated Circuits, Memory Testing, Single Event Upset (SEU)and Negative Bias Temperature Instability (NBTI) Aware, Design of IntegratedCircuits and Memories, and emerging technologies, such as memristors.

E. Brum graduated in Computer Engineering at Pontifical Catholic University of Rio Grande do Sul (PUCRS) in 2021. His fields of interest include test and reliability of FinFET-based SRAMs and emerging technologies.

S. Menzel was born in Bremen, Germany. He received the Diploma degree and the Ph.D. degree (summa cum laude) in electrical engineering from RWTH Aachen University, Aachen, Germany, in 2005 and 2012, respectively. His current research interests include simulation and physical modeling of resistive random-access memory devices

S. Hamdioui (Senior Member, IEEE) received the M.S.E.E. and Ph.D. degrees (Hons.) from the Delft University of Technology (TU Delft), The Netherlands. He was with Intel Corporation, CA, USA, with Philips Semiconductors Research and Development, Crolles, France, and with Philips/NXP Semiconductors, Nijmegen, The Netherlands. $\mathrm{He}$ is currently the Chair Professor of dependable and emerging computer technologies, the Head of the Computer Engineering Laboratory (CE-Lab), and the Head of the Quantum and Computer Engineering Department, TU Delft. He is also the Co-Founder and the CEO of Cognitive- IC, a start-up focusing on hardware dependability solutions. His research interests include two domains: dependable CMOS nanocomputing (including testability, reliability, and hardware security) and emerging technologies and computing paradigms (including memristors for logic and storage, in- memory-computing, and neuromorphic computing).

T. Gemmeke received the M.S. and Ph.D. degrees in electrical engineering from RWTH Aachen University, Aachen, Germany, in 1998 and 2006, respectively. In 2004, he transitioned to IBM's R\&D Organization, Böblingen, Germany, targeting high performance processors. In 2007, he joined the former start-up Aquantia Corporation conceiving energy efficient PHY solutions for the 10GBase-T over copper standard. In 2011, he was the Technical Lead of the Digital Design Team, Holst Centre/IMEC, Eindhoven, The Netherlands, focusing on ultralow-power design of wearable sensor nodes. Since 2017, he has been a Full Professor with the Chair of integrated digital system with RWTH Aachen University. His research interests include the design of digital systems considering all entry levels from algorithmic optimization down to the physically oriented design. 